\title{
Serum progranulin levels are elevated in dermatomyositis patients with acute interstitial lung disease, predicting prognosis
}

Atsushi Tanaka', Hiroshi Tsukamoto ${ }^{1 *}$, Hiroki Mitoma ${ }^{1}$, Chikako Kiyohara², Naoyasu Ueda ${ }^{1}$, Masahiro Ayano ${ }^{1}$, Shun-ichiro Ohta ${ }^{1}$, Yasutaka Kimoto ${ }^{3}$, Mitsuteru Akahoshi' ${ }^{1}$, Yojiro Arinobu' ${ }^{1}$, Hiroaki Niiro ${ }^{1}$, Yoshifumi Tada ${ }^{4}$, Takahiko Horiuchi $^{3}$ and Koichi Akashi ${ }^{1}$

\begin{abstract}
Introduction: Progranulin (PGRN), a pleiotropic growth factor, has emerged as an immunoregulatory molecule. Because the roles of PGRN in dermatomyositis (DM) are still unknown, we investigated whether serum PGRN levels are associated with disease activity and prognosis in DM patients, particularly in those with DM complicated with interstitial lung disease (ILD).

Methods: The serum levels of PGRN were measured by enzyme-linked immunosorbent assay in patients with DM ( $n=57$; acute/subacute interstitial pneumonia (A/SIP): $n=17$, chronic interstitial pneumonia (CIP): $n=24$, without ILD: $n=16$ ), polymyositis (PM, $n=21$; including 6 with ILD) and normal healthy controls (NHCs, $n=60)$. We assessed the correlation between the serum PGRN levels and the activity indexes of ILD or prognosis in DM patients with ILD.

Results: Serum PGRN levels were significantly higher in DM patients than in PM patients $(P=0.0025)$ and in NHCS $(P<0.0001)$. In DM patients, the levels were significantly higher in patients with $\mathrm{A} / \mathrm{SIP}$ than in those with $\mathrm{CIP}(P<0.0001)$ or without ILD ( $P=0.0003)$. The serum PGRN levels in DM patients with ILD significantly correlated with serum ferritin $\left(r_{S}=0.77, P<0.0001\right)$, lactate dehydrogenase $\left(r_{S}=0.54, P=0.0003\right)$ and $C$-reactive protein $\left(r_{S}=0.48, P=0.0015\right)$ levels. Moreover, in DM patients with ILD, the cumulative survival rate for 6 months was significantly lower in the group with serum PGRN levels $\geq 200 \mathrm{ng} / \mathrm{ml}(67 \%)$ than in the group with serum PGRN levels $<200 \mathrm{ng} / \mathrm{ml}(100 \%)(P=0.0009)$.
\end{abstract}

Conclusions: Serum PGRN is associated with disease activity and prognosis of DM with ILD. PGRN may play a role in the pathogenesis of DM and could be a useful biomarker.

\section{Introduction}

Dermatomyositis (DM) is a systemic autoimmune and inflammatory disease that involves not only the muscle and skin but also several other organs, such as the lungs, heart and joints [1]. Interstitial lung disease (ILD) is the most common internal organ manifestation, and it affects the prognosis of DM patients. ILD is classified into two subsets: acute/subacute interstitial pneumonia (A/SIP) and chronic interstitial pneumonia (CIP) [2,3]. A/SIP is often complicated with clinically amyopathic DM (CADM) $[4,5]$, which shows the typical skin manifestations of DM

\footnotetext{
* Correspondence: tsukamot@intmed1.med.kyushu-u.ac.jp

'Department of Medicine and Biosystemic Science, Kyushu University Graduate School of Medical Sciences, 3-1-1 Maidashi, Higashi-ku, Fukuoka 812-8582, Japan

Full list of author information is available at the end of the article
}

but has no or little evidence of clinical myositis [6]. A/SIP complicated with CADM is life-threatening and shows a rapidly progressive pattern, with a 6-month survival rate of $40.8 \%$, irrespective of intensive therapy [5]. Combination therapy with prednisolone (PSL), cyclosporine A (CsA) and intravenous pulse cyclophosphamide (IVCY) is more effective for A/SIP with DM than PSL plus one immunosuppressive agent [7]. Therefore, diagnosis and evaluation of ILD is very important to determine the treatment strategy when DM is diagnosed [8-10]. Although several biomarkers that may reflect inflammatory activity of lung have been utilized, such as ferritin [11-14] and Krebs von den Lungen-6 (KL-6) [15-19], there are no established serum biomarkers for DM-associated ILD.

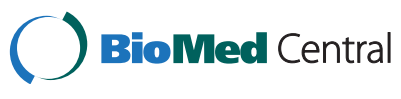

(C) 2015 Tanaka et al.; licensee BioMed Central. This is an Open Access article distributed under the terms of the Creative Commons Attribution License (http://creativecommons.org/licenses/by/4.0), which permits unrestricted use, distribution, and reproduction in any medium, provided the original work is properly credited. The Creative Commons Public Domain Dedication waiver (http://creativecommons.org/publicdomain/zero/1.0/) applies to the data made available in this article, unless otherwise stated. 
Progranulin (PGRN) [GenBank:NC_000017] is an extracellular glycoprotein containing seven and one-half repeats of cysteine-rich motifs. PGRN is proteolytically cleaved by extracellular proteases, such as proteinase 3 (PR3) and elastase, into granulin (GRN) [20] that range from 6 to $25 \mathrm{kDa}$. PGRN is abundantly expressed in rapidly cycling epithelial cells, leukocytes, chondrocytes and neurons [21], and its expression level is at steady state [22]. PGRN plays a critical role in early embryogenesis [22], wound healing [23], maintenance of neuronal survival [24] and tumorigenesis [21]. Particularly, macrophage-derived PGRN is a key regulatory factor in the processes of inflammation and wound healing $[23,25]$. Recent mouse studies show that mice unable to convert PGRN into GRN due to lack of both elastase and PR3 cannot show inflammation in response to injection of immune complexes [26]. These data indicate that PGRN is synthesized by macrophages and is cleaved into GRNs by elastase in tissues to enhance inflammation. Moreover, PGRN and/or GRN act as a soluble cofactor for Toll-like receptor 9 (TLR9) signaling and enhance it [27]. We previously reported that serum PGRN levels are significantly elevated in systemic lupus erythematosus (SLE) patients in parallel with disease activities and that PGRN may have a role in the pathogenesis of SLE, partly by enhancing the TLR9 signaling and interleukin (IL)-6 production [28]. Although macrophage activation is considered to underlie the pathogenesis of A/SIP with DM [11-14] and TLR9 and IL-6 is associated with DM [29-35], the roles of PGRN in DM are still unknown.

Here, we show that serum PGRN levels are significantly elevated in DM patients, particularly those with $\mathrm{A} / \mathrm{SIP}$, and are associated with disease activity and prognosis of DM patients with ILD. PGRN may have a role in the pathogenesis of DM.

\section{Methods \\ Patients}

We performed a cross-sectional study of patients who were treated for DM or polymyositis (PM) at Kyushu University Hospital, Saga University Hospital and Japanese Red Cross Fukuoka Hospital between 2002 and 2013. Fifty-seven Japanese patients with DM and twenty-one patients with PM were enrolled, and sera were obtained from these patients. All of the patients fulfilled the criteria of Bohan and Peter [36] for DM or PM or criteria for CADM [37]. We defined active DM and PM patients who required the initiation or reinforcement of treatment (overall disease activity). We excluded patients with other autoimmune and infectious diseases. Each patient completed a standardized medical history, including drug use, and was given a physical examination. The information obtained from the medical records of the patients included demographic data, such as age, sex, clinical manifestations of DM and laboratory values. Serological profiling of each patient, including white blood cells (WBCs), lymphocytes, hemoglobin $(\mathrm{Hb})$, platelets (Plt), liver enzymes (aspartate aminotransferase (AST), alanine aminotransferase (ALT), $\gamma$-glutamyl transpeptidase ( $\gamma$-GTP)), creatine kinase $(\mathrm{CK})$, lactate dehydrogenase (LDH), ferritin, KL-6, C-reactive protein (CRP), anti-nuclear antibody (ANA) and anti-Jo-1 antibody, was performed using the standard methods. The serum samples from the patients with active DM were acquired before the initiation or reinforcement of treatment, and the samples from the patients with inactive DM were acquired during regular hospital visits. The samples were stored at $-20^{\circ} \mathrm{C}$. The active DM patients were treated with corticosteroids or immunosuppressive drugs after the completion of these evaluations. The sera obtained from five DM patients with ILD were reevaluated after ILD was ameliorated by treatment. Control sera were obtained from healthy staff members $(n=60)$ at Kyushu University Hospital. This study was approved by the ethics committees of Kyushu University Hospital, Saga University Hospital and Japanese Red Cross Fukuoka Hospital, and the principles of the Declaration of Helsinki were followed throughout the study. Informed consent was obtained from all participants.

\section{Measurement of PGRN}

The serum PGRN levels were determined using enzymelinked immunosorbent assay (ELISA) kits (R\&D Systems, Minneapolis, MN, USA) according to the manufacturer's protocol. Briefly, calibrators, control sera and patients' sera (stored samples) were diluted and incubated with a mouse monoclonal antibody against PGRN adsorbed onto the microtiter plate wells. After washing, a mouse monoclonal antibody against PGRN conjugated to horseradish peroxidase was added, followed by a second washing step and the addition of tetramethylbenzidine substrate. The intensity of the blue color developed was in proportion to the amount of PGRN bound in the initial step. The reaction was terminated by the addition of $2 \mathrm{~N}$ sulfuric acid. The absorbance was measured in a microtiter plate reader (Thermo Fisher Scientific, Waltham, MA, USA) and converted into nanograms per milliliter by plotting the values against the PGRN titer of the calibrators/standards given by the manufacturer. The assay range was 1.56 to $100 \mathrm{ng} / \mathrm{ml}$.

\section{Classification of ILD}

ILD was assessed by radiography and high-resolution computed tomography of the chest. ILD with DM was divided into two forms: A/SIP and CIP. A/SIP is defined as a rapidly progressive ILD within 3 months from the onset of symptoms and radiographically diffuse alveolar damage pattern or nonspecific interstitial pneumonias (NSIP). CIP is defined as an asymptomatic, non-rapidly progressive ILD or slowly progressive ILD over the 
course of 3 months and radiographically confirmed NSIP or bronchiolitis obliterans with organizing pneumonia pattern, according to the guidelines of the International Consensus Statement of idiopathic Pulmonary Fibrosis of the American Thoracic Society and the European Respiratory Society [38]. The designation of A/SIP and CIP was made blindly without knowledge of the laboratory results.

\section{Statistical analysis}

The differences between two groups were analyzed using the Mann-Whitney $U$ test. Fisher's exact test was used for comparison of frequencies. The Steel-Dwass test was used for multiple comparisons among all groups. The relationships between PGRN levels and other continuous variables were analyzed using Spearman's rank correlation. Receiver operating characteristic (ROC) analyses were performed for a mathematical expression of different serum PGRN levels as cutoff points. The cumulative survival rate was calculated using the Kaplan-Meier test. The log-rank test was also used to compare survival. $P$-values less than 0.05 were considered significant. All tests were two-tailed. All analyses were performed using JMP statistical software (SAS Institute, Cary, NC, USA).

\section{Results}

\section{Serum PGRN levels were markedly elevated in patients with DM}

Of the 57 patients with DM enrolled in the present study, 49 were women, and 8 were men (active: $\mathrm{n}=40$, inactive: $\mathrm{n}=17$ ). These patients ranged in age from 30 to 87 years (median age: 53 years). Of the 21 patients with PM, 16 were women, and 5 were men (active: $\mathrm{n}=11$, inactive: $\mathrm{n}=10$ ). These patients ranged in age from 15 to 85 years (median age: 53 years). Among the NHCs, 51 were women, and 9 were men. They ranged in age from 20 to 59 years (median age: 32 years). There were significant differences between patients with DM or PM and NHCs in terms of age. However, there was not a significant correlation between serum PGRN levels and age in NHCs.

To investigate the association of PGRN with DM, we first compared serum PGRN levels among 57 patients with DM, 21 patients with PM and 60 NHCs by using ELISA (Figure 1). Serum PGRN levels in NHCs were always within the range of 35 to $70 \mathrm{ng} / \mathrm{ml}$ and distributed normally. Serum PGRN levels in patients with PM (median: $60 \mathrm{ng} / \mathrm{ml}$ ) were significantly higher than in healthy controls $(48 \mathrm{ng} / \mathrm{ml})(P<0.0001)$. Strikingly, serum PGRN levels in patients with DM (median: $109 \mathrm{ng} / \mathrm{ml}$ ) were significantly and markedly higher than in PM patients $(P=0.0025)$ and in healthy controls $(P<0.0001)$.

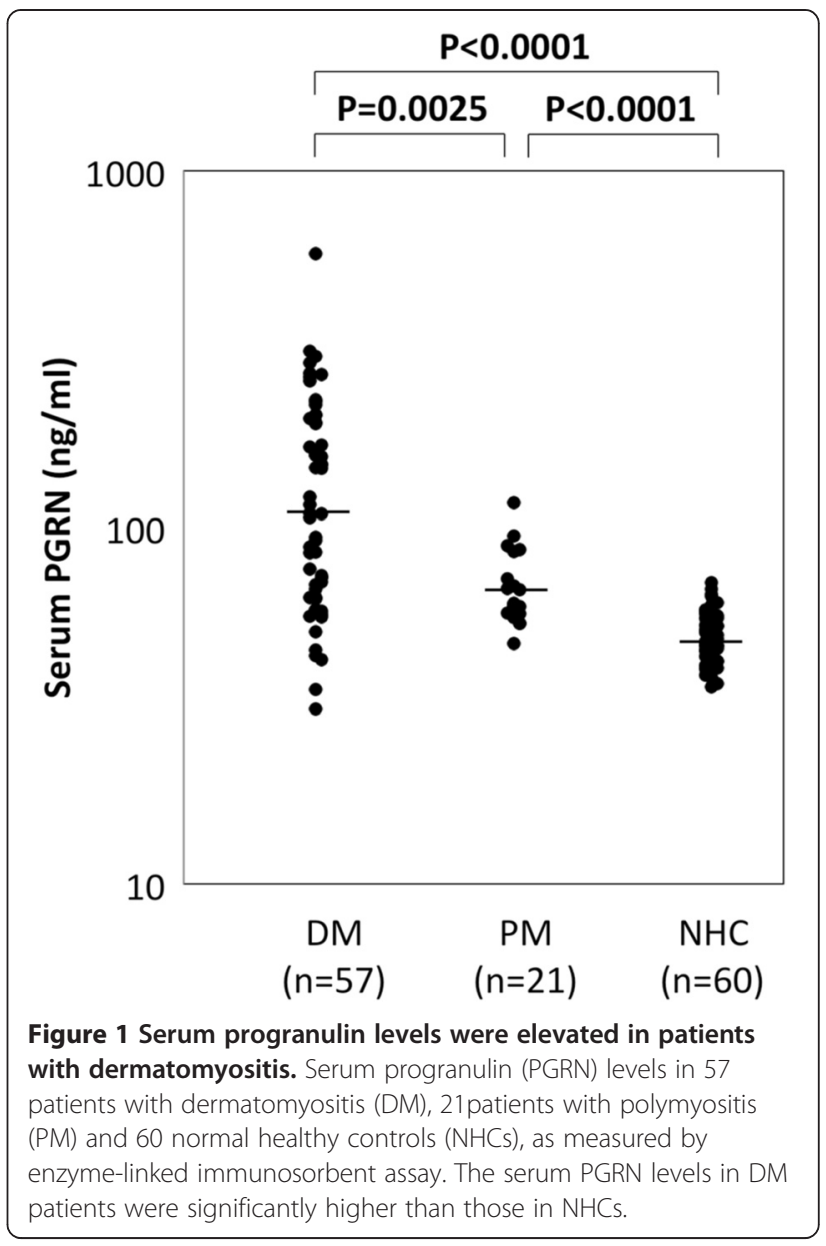

Serum PGRN levels were elevated in DM patients with A/SIP Because DM is occasionally complicated with ILD [8-10], we next compared serum PGRN levels among DM patients with A/SIP ( $\mathrm{n}=17)$, those with CIP ( $\mathrm{n}=24)$ and those without ILD $(\mathrm{n}=16)$ (Figure 2). Serum PGRN levels in DM patients with A/SIP (median: $228 \mathrm{ng} / \mathrm{ml}$ ) were significantly higher than in DM patients with CIP $(75 \mathrm{ng} / \mathrm{ml}, P<0.0001)$ or without ILD (79 ng/ml, $P=0.0003$ ). There was no significant difference between serum PGRN levels in DM patients with CIP and those without ILD $(P=0.8382)$. However, serum PGRN levels in DM patients with CIP or without ILD were significantly higher than in NHCs ( $P<0.0001$ and $P=0.005$, respectively; data not shown).

\section{Comparison of clinical manifestations between DM patients with A/SIP and without A/SIP}

To evaluate clinical manifestations other than serum PGRN levels, we compared those between DM patients with A/SIP and without A/SIP (Table 1). There were no significant differences among all the subsets in terms of age and sex. The duration between the onset and evaluation in DM patients with A/SIP was significantly 


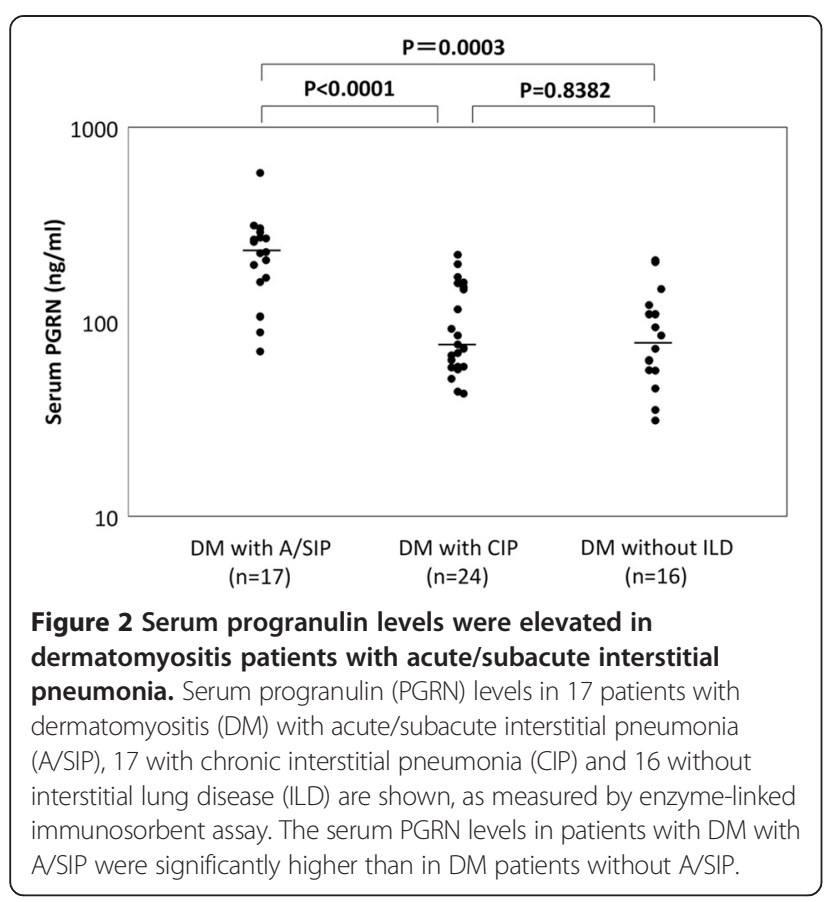

Table 1 Comparison of clinical manifestations between dermatomyositis patients with acute/subacute interstitial pneumonia and without acute/subacute interstitial pneumonia ${ }^{a}$

\begin{tabular}{|c|c|c|c|}
\hline Variables & DM with A/SIP & DM with CIP & DM without ILD \\
\hline Number of patients & 17 & 24 & 16 \\
\hline Age, yr & 54.0 (36 to 65$)$ & 54.5 (19 to 78$)$ & 36 (15 to 85$)$ \\
\hline Female, n (\%) & $14(82.4)$ & $20(83.3)$ & $15(93.8)$ \\
\hline Duration, mo & 2 (1 to 19$)$ & $33(1 \text { to } 148)^{* * *}$ & $11(1 \text { to } 237)^{* *}$ \\
\hline Muscle weakness, n (\%) & $4(23.5)$ & $15(62.5)^{*}$ & $15(94.1)^{* * *}$ \\
\hline WBCs, count/ml & $5,200(2,900$ to 12,300$)$ & $8,165(2,900$ to 14,990$)$ & $7,590(3,540$ to 11,580$)$ \\
\hline $\mathrm{Hb}, \mathrm{g} / \mathrm{dl}$ & 11.6 (7.9 to 13.9$)$ & 12.8 (10 to 15.4$)$ & 12.5 (9.8 to 15.3$)$ \\
\hline$P \mid t, \times 10^{5} / \mu l$ & $22.2(14.9$ to 34.5$)$ & 23.0 (12 to 38.8$)$ & 20.6 (6.2 to 37.7$)$ \\
\hline CK, IU/L & 209 (33 to 866) & $1,289$ (16 to 12,300$)$ & 568 (51 to 2,646$)$ \\
\hline AST, IU/L & 74 (31 to 219) & $30(14 \text { to } 657)^{* *}$ & $44(12 \text { to } 132)^{*}$ \\
\hline ALT, IU/L & $42(19$ to 111$)$ & $20(11 \text { to } 301)^{*}$ & 31 (10 to 150$)$ \\
\hline Y-GTP, IU/L & 43 (16 to 346$)$ & $24(10$ to 131$)$ & 26 (10 to 165$)$ \\
\hline LDH, IU/L & 392 (225 to 957) & $273(186 \text { to } 1,975)^{*}$ & $328(157 \text { to } 489)^{*}$ \\
\hline Ferritin, ng/ml & 875 (251 to 2,171$)$ & $88(16 \text { to } 4,853)^{* * *}$ & $105(39 \text { to } 400)^{* * *}$ \\
\hline $\mathrm{KL}-6, \mathrm{U} / \mathrm{ml}$ & 959 (338 to 2,048) & $525(188 \text { to } 1,802)^{*}$ & $224(125 \text { to } 459)^{* * *}$ \\
\hline PGRN, ng/ml & 228 (70 to 586) & $75(43 \text { to } 221)^{* * *}$ & 79 (31 to 207) \\
\hline CRP, mg/dl & 0.86 (0.03 to 2.97$)$ & $0.24(0.03 \text { to } 3.66)^{* *}$ & $0.08(0.01 \text { to } 0.9)^{* * *}$ \\
\hline ANA > 160, n (\%) & $1(5.9)$ & $2(8.3)$ & $5(31.3)$ \\
\hline Anti-Jo-1, n (\%) & $0(0)$ & $4(16.7)$ & $0(0)$ \\
\hline
\end{tabular}

${ }^{a}$ ANA, Anti-nuclear antibody; A/SIP, Acute/subacute interstitial pneumonia; ALT, Alanine aminotransferase; AST, Aspartate aminotransferase; CIP, Chronic interstitial pneumonia; CK, Creatine kinase; CRP, C-reactive protein; DM, Dermatomyositis; $\gamma$-GTP, $\gamma$-glutamyl transpeptidase; Hb, Hemoglobin; ILD, Interstitial lung disease; KL-6, Krebs von den Lungen-6; LDH, Lactate dehydrogenase; PIt, Platelets; WBC, White blood cell. The values of age, duration, CK, AST, ALT, Y-GTP, LDH, ferritin, KL-6, PGRN and CRP indicate median (range). ${ }^{*} P<0.05,{ }^{* *} P<0.01,{ }^{* *} P<0.001$ compared with DM with A/SIP using the Mann-Whitney $U$ test or Fisher's exact test. with disease activities of ILD

We next tested whether serum PGRN levels in DM patients with ILD correlate with conventional serologic parameters for disease activity of ILD (Table 2). Serum PGRN levels showed a significantly positive correlation with serum levels of $\mathrm{LDH}\left(r_{\mathrm{S}}=0.54, P=0.0003\right)$, ferritin $\left(r_{\mathrm{S}}=0.77, P<0.0001\right)$ and $\mathrm{CRP}\left(r_{\mathrm{S}}=0.48, P=0.0015\right)$. shorter than in those without A/SIP. The frequency of ratio of CADM patients was significantly higher in DM
patients with A/SIP than in those without it. WBCs and in those without it. Although CK levels showed no significant difference among all the subsets, AST and ALT SIP than in those with CIP and wh patients with A/ of LDH, ferritin, KL- 6 and CRP, which are used as serologic parameters for disease activity of ILD, showed sig-
nificant difference between DM patients with A/SIP and logic parameters for disease activity of ILD, showed sig-
nificant difference between DM patients with A/SIP and positivity was not significantly different among all the subsets.

\section{Serum PGRN levels in DM patients with ILD correlated}

with A/SIP than in those without it, indicating that the $\mathrm{Hb}$ tended to be lower in DM patients with A/SIP than levels were significantly higher in DM patients with A/ SIP than in those with CIP and without ILD. The levels those without A/SIP. The frequency of ANA or anti-Jo-1 
Table 2 Serum progranulin levels in dermatomyositis patients with interstitial lung disease correlate with disease activity of interstitial lung disease ${ }^{a}$

\begin{tabular}{lll}
\hline Variables & $\boldsymbol{r}_{\mathbf{S}}$ & $\boldsymbol{P}$-value \\
\hline PGRN vs LDH & 0.54 & 0.0003 \\
PGRN vs ferritin & 0.77 & $<0.0001$ \\
PGRN vs KL-6 & 0.26 & 0.1034 \\
PGRN vs CRP & 0.48 & 0.0015
\end{tabular}

${ }^{a}$ CRP, C-reactive protein; KL-6, Krebs von den Lungen-6; LDH, Lactate dehydrogenase; PGRN, Progranulin; $r_{\mathrm{S}}$, Correlation coefficient established employing Spearman's correlation coefficients. Correlations between the serum PGRN levels and LDH, ferritin, KL-6 and CRP are shown, as measured in 41 DM patients with ILD.

However, there was not a significant correlation between serum levels of PGRN and KL-6 $\left(r_{\mathrm{S}}=0.26, P=0.1034\right)$. In five DM patients with active ILD that had subsided with treatment, serum PGRN levels decreased in all of them (Figure 3). These results indicate that serum PGRN levels reflect disease activity of ILD in DM patients.

\section{Treatment}

The frequency of treatment with PSL alone was fairly low in patients with DM with A/SIP, whereas the frequency of treatment with immunosuppressive agents in addition to PSL was significantly higher in DM patients with A/SIP than in DM patients without ILD $(P=0.0004)$ (Table 3$)$. Immunosuppressive agents used in patients with DM with

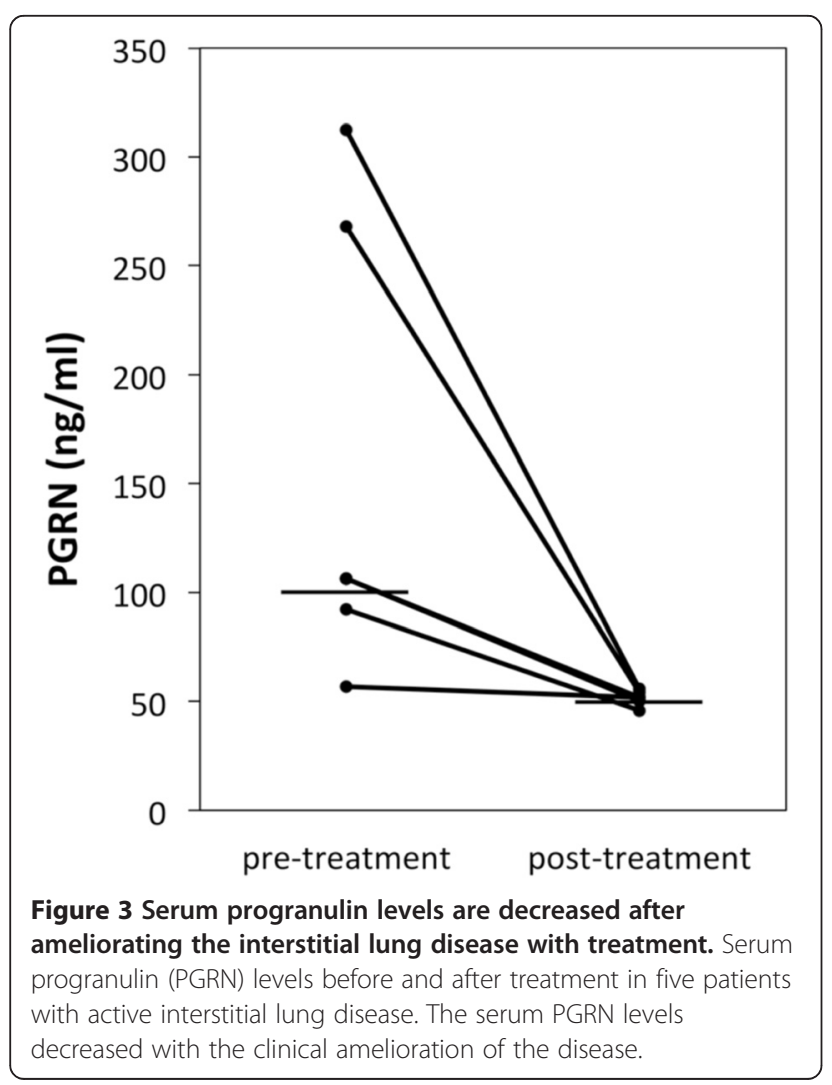

Table 3 Treatment in each group ${ }^{a}$

\begin{tabular}{llll}
\hline Treatments & DM with A/SIP & $\begin{array}{l}\text { DM with } \\
\text { CIP }\end{array}$ & $\begin{array}{l}\text { DM without } \\
\text { ILD }\end{array}$ \\
\hline Number of patients & 17 & 24 & 16 \\
PSL or other agent alone, $n(\%)$ & $3(18)$ & $8(33)$ & $12(75)^{* *}$ \\
PSL & 3 & 7 & 11 \\
CNI & 0 & 1 & 0 \\
AZA & 0 & 0 & 1 \\
PSL + other agents, n (\%) & $14(82)$ & $16(67)$ & $3(19)^{* *}$ \\
CNI & 9 & 13 & 1 \\
MTX & 0 & 0 & 2 \\
IVCY & 0 & 1 & 0 \\
CNI + MZB & 0 & 1 & 0 \\
CNI + IVCY & 3 & 0 & 0 \\
HSCT & 2 & 1 & 0
\end{tabular}

${ }^{a} \mathrm{~A} / \mathrm{SIP}$, Acute/subacute interstitial pneumonia; AZA, Azathioprine; CIP, Chronic interstitial pneumonia; CNI, Calcineurin inhibitor (cyclosporine or tacrolimus); DM, Dermatomyositis; HSCT, Hematopoietic stem cell transplant; ILD, Interstitial lung disease; IVCY, Intravenous pulse cyclophosphamide; MTX, Methotrexate; MZB, Mizoribine; PSL, Prednisolone. ${ }^{* *} P<0.01$ compared with DM with A/SIP using Fisher's exact test.

A/SIP were calcineurin inhibitors (CNIs: CsA or tacrolimus) for nine patients and the combination therapy with CNI plus IVCY for three patients. We performed hematopoietic stem cell transplants (HSCTs) for two refractory DM patients with A/SIP, irrespective of the combination therapy with PSL, CNI plus IVCY, and could rescue them [39].

\section{Serum PGRN levels in DM patients with ILD were associated with prognosis}

Because DM with ILD is occasionally life-threatening $[4,5,7]$, in order to define the optimal cutoff point with the highest diagnostic accuracy in terms of survival, we performed ROC curve analysis for distinct serum PGRN levels. The highest area under the curve was calculated for a baseline serum PGRN level of $200 \mathrm{ng} / \mathrm{ml}$. The two subsets were classified by serum PGRN level of $200 \mathrm{ng} / \mathrm{ml}$. The cumulative survival rates for 6 months were $66.7 \%$ and $100 \%$ in the group with serum PGRN levels $\geq 200 \mathrm{ng} / \mathrm{ml}$ ( $\mathrm{n}=12$ ), including two patients treated with HSCT, and the group with serum PGRN levels <200 ng/ml ( $\mathrm{n}=29$ ) in DM with ILD, respectively (Figure 4). The cumulative survival rate was significantly lower in the group with serum PGRN levels $\geq 200 \mathrm{ng} / \mathrm{ml}$ than in the group with serum PGRN levels $<200 \mathrm{ng} / \mathrm{ml}(P=0.0009)$. These results suggest that baseline serum PGRN levels predict the prognosis of DM patients with ILD.

\section{Discussion}

To our knowledge, this is the first study to show that PGRN levels were significantly elevated in sera of DM patients, in particular those with A/SIP, and these 


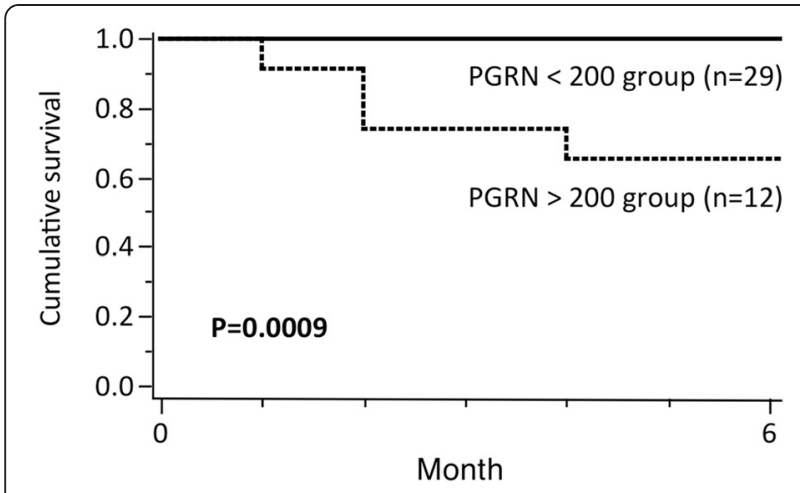

Figure 4 Serum progranulin levels in dermatomyositis patients with interstitial lung disease were associated with prognosis. The cumulative survival rate for 6 months in the serum progranulin (PGRN) $<200 \mathrm{ng} / \mathrm{ml}$ group and the $\geq 200 \mathrm{ng} / \mathrm{ml}$ group of patients with dermatomyositis with interstitial lung disease are shown. The cumulative survival rate was calculated using the Kaplan-Meier test. The log-rank test was also used to compare survival. The cumulative survival rate was significantly lower in the group with serum PGRN levels $\geq 200 \mathrm{ng} / \mathrm{ml}$ than in the group with serum PGRN levels $<200 \mathrm{ng} / \mathrm{ml}$.

concentrations were associated with the disease activity and prognosis of DM patients with ILD.

Consistent with previous reports $[11,12]$, the elevation of transaminases and hyperferritinemia were observed in DM with A/SIP in the present study. In addition, WBC and $\mathrm{Hb}$ tended to be lower in $\mathrm{DM}$ patients with $\mathrm{A} / \mathrm{SIP}$ than in those without it. These facts suggest that macrophages are substantially activated and cause injury to the lungs and the liver in DM patients with A/SIP. Actually, the accumulation of ferritin-producing macrophages has been shown in a CADM-related acute interstitial pneumonia autopsy case [40]. Because PGRN is highly expressed in macrophages [22], elevation of serum PGRN levels in $\mathrm{DM}$ with A/SIP also reflects macrophage activation as that of ferritin.

In the present study, other than DM, we also measured concentrations of serum PGRN in patients with PM ( $\mathrm{n}=21$ ), including 6 with ILD (median: $59 \mathrm{ng} / \mathrm{ml}$ ) and 15 with systemic sclerosis (median: $49 \mathrm{ng} / \mathrm{ml}$ ) (same levels in NHCs), including 13 with ILD (median: $49 \mathrm{ng} / \mathrm{ml}$ ). In our data, although sera from PM patients contained significantly elevated PGRN compared with NHCs, DM patients' sera had much higher levels of PGRN, especially among DM patients who also had A/SIP. This difference may occur because activated macrophages appeared especially in DM patients with A/SIP. Although we could not recruit acute respiratory distress syndrome (ARDS) patients into this study, ARDS could be an additional control to determine whether PGRN is a more general marker of acute respiratory inflammation.

PGRN is reported to be important in the initiation of inflammation by recruiting fibroblasts, macrophages and neutrophils to the site of inflammation [23]. PGRN is also converted to GRN by the elastase that is produced by leukocytes and other cells [41], however, and recent mouse studies have shown that GRN possesses inflammatory functions $[25,26,42]$. Thus, PGRN may be converted to GRN in the lungs and may be associated with the early phase of pathogenesis in DM with A/SIP. However, it is difficult to investigate the function of GRN in human studies, because we cannot measure GRNs at this moment.

Serum PGRN levels showed a significantly positive correlation with serum levels of LDH $\left(r_{\mathrm{S}}=0.54, P=0.0003\right)$ (Table 2) and ferritin $\left(r_{\mathrm{S}}=0.77, P<0.0001\right)$. However, there was no significant correlation between serum levels of PGRN and those of KL-6, another biomarker for ILD. Recent studies suggest serum ferritin levels as a marker for severity of acute progressive ILD in DM and CADM patients [11-14]. The serum ferritin concentrations in CADM patients with A/SIP are already elevated in early stages of the disease, before progression of ILD [11]. LDH and surfactant protein D are released into the blood when pulmonary cells are damaged with inflammation [43]. KL6 rather reflects the regeneration and proliferation of pneumocytes after the damage of the pulmonary tissue [44]. Thus, PGRN could also be an acute marker for ILD as well as ferritin and LDH.

When we compared clinical manifestations between DM patients with A/SIP and without A/SIP, the frequency of CADM was significantly higher in those with A/SIP (76.5\%) than in those with CIP (37.5\%) and in those without ILD (5.9\%). CADM may be a useful indicator for the occurrence of A/SIP. The levels of CRP showed significant differences between DM patients with $\mathrm{A} / \mathrm{SIP}$ and those without and correlated with serum PGRN levels $\left(r_{\mathrm{S}}=0.48, P=0.0015\right)$ (Table 2). We previously reported that serum PGRN levels are elevated in SLE patients and that PGRN stimulated IL- 6 production via TLR9 [28]. Upregulated IL-6 production stimulated by PGRN $[27,28,45]$ may be related to elevation of CRP in DM patients with A/SIP. Serum IL-6 levels were significantly higher in dead than alive CADM patients with ILD [33] and were reported to be candidate biomarkers for disease activity in DM [32] and SLE [46]. Because TLR9 is highly expressed in DM [29-31] and SLE [47], upregulated IL- 6 production stimulated by PGRN via TLR9 may be a common pathogenesis of DM and SLE. However, the proposed role of PGRN signaling via TLR9 in DM must be directly demonstrated in future studies. Elevated serum PGRN levels have been noted for a number of other pathological conditions [48]. So, PGRN is most likely not a disease-specific marker, but rather is probably a marker of immune activation.

Most of the DM patients with CIP were treated with PSL and immunosuppressive agents. HSCT was performed 
for one DM patient with CIP and a refractory skin ulcer. In contrast, several patients with A/SIP required combination therapy with PSL, CNI and IVCY (and HSCTs). This distinction might be responsible for cellular phenotypes affecting the pathogenesis of ILD.

The cumulative survival rate was significantly lower in the group with serum PGRN levels $\geq 200 \mathrm{ng} / \mathrm{ml}$ than in the group with serum PGRN levels $<200 \mathrm{ng} / \mathrm{ml}(P=0.0009)$ (Figure 4). These data indicate that baseline serum PGRN levels can predict survival. The group with serum PGRN levels $\geq 200 \mathrm{ng} / \mathrm{ml}$ included two refractory patients with A/SIP treated with HSCTs, which saved their lives. If we had not been able to rescue them, the survival rate in the group with serum PGRN levels $\geq 200 \mathrm{ng} / \mathrm{ml}$ would have been lower. It is inferred from our findings that the intensive combination therapy with various immunosuppressive agents should be chosen for DM patients with ILD showing high serum PGRN levels, especially $\geq 200 \mathrm{ng} / \mathrm{ml}$.

We must acknowledge some limitations of this study. The sample size of our study was small. Statistical tests usually require a larger sample size to justify that the effect did not happen by chance alone. Moreover, owing to the study's cross-sectional design, it is difficult to establish the exact and definite causal relationships, except the association between PGRN and development of ILD with DM, on the basis of the collected data. There were potential confounding effects of medication use on serum PGRN levels in patients with inactive DM with CIP and without ILD. Last, to fully validate PGRN as a predictive biomarker, we need to show that elevated PGRN levels predate clinical respiratory decompensation.

\section{Conclusions}

In conclusion, this pilot study demonstrates that the serum PGRN levels were elevated in patients with DM, in particular those with A/SIP, and were correlated with disease activity and prognosis of DM patients with ILD. PGRN could be a useful biomarker for disease activity and a predictor of survival in DM patients with ILD. These findings of the study will provide new insights into the pathogenesis as well as the therapy of DM, and will shed new light on the dysregulation of the immune system in autoimmune diseases. Further studies are required to reveal more precisely the mechanisms of PGRN in human autoimmune diseases.

\footnotetext{
Abbreviations

A/SIP: Acute/subacute interstitial pneumonia; ALT: Alanine aminotransferase; ANA: Anti-nuclear antibody; ARDS: Acute respiratory distress syndrome; AST: Aspartate aminotransferase; AZA: Azathioprine; CADM: Clinically amyopathic dermatomyositis; CIP: Chronic interstitial pneumonia; CK: Creatine kinase; CNI: Calcineurin inhibitor; CRP: C-reactive protein; CSA: Cyclosporine A; DM: Dermatomyositis; ELISA: Enzyme-linked immunosorbent assay; $\gamma$-GTP: $\gamma$-glutamyl transpeptidase; GRN: Granulin; Hb: Hemoglobin; HSCT: Hematopoietic stem cell transplantation; IL: Interleukin; ILD: Interstitial lung disease; IVCY: Intravenous pulse cyclophosphamide; KL-6: Krebs von den Lungen-6; LDH: Lactate
}

dehydrogenase; MTX: Methotrexate; MZB: Mizoribine; NHC: Normal healthy control; NSIP: Nonspecific interstitial pneumonia; PGRN: Progranulin; Plt: Platelet; PM: Polymyositis; PR3: Proteinase 3; PSL: Prednisolone; ROC: Receiver operating characteristic; SLE: Systemic lupus erythematosus; TLR: Toll-like receptor; WBC: White blood cell.

\section{Competing interests}

The authors declare that they have no competing interests.

\section{Authors' contributions}

AT performed the ELISA experiments, the statistical analysis and drafted the manuscript. HT, HM, TH and KA designed the study and helped to draft and revise the manuscript. CK performed the important statistical analysis and helped to revise the manuscript. MAy and SO assisted in conducting the ELISA experiments, contributed to data analysis and helped to draft the manuscript. NU, YK, MAK, YA, HN and YT contributed to the acquisition, analysis and interpretation of clinical data and drafted the manuscript. All authors read and approved the final manuscript.

\section{Acknowledgements}

This work was supported in part by grants from the Ministry of Health, Labor and Welfare (H24-Jitsuyoka-Kokusai-004) and from the Ministry of Education, Science, Sports, and Culture of Japan (24591465). We are grateful to the patients and volunteers for their participation in this study and thank all the rheumatologists at the Kyushu University Hospital, Saga University Hospital and Japanese Red Cross Fukuoka Hospital who participated in this study.

\section{Author details}

${ }^{1}$ Department of Medicine and Biosystemic Science, Kyushu University Graduate School of Medical Sciences, 3-1-1 Maidashi, Higashi-ku, Fukuoka 812-8582, Japan. ${ }^{2}$ Department of Preventive Medicine, Kyushu University Graduate School of Medical Sciences, Fukuoka 812-8582, Japan. ${ }^{3}$ Department of Internal Medicine, Kyushu University Beppu Hospital, Oita 874-0838, Japan. ${ }^{4}$ Department of Rheumatology, Faculty of Medicine, Saga University, Saga 849-8501, Japan.

Received: 18 June 2014 Accepted: 27 January 2015

Published online: 10 February 2015

\section{References}

1. Callen J. Dermatomyositis Lancet. 2000;355:53-7.

2. Ito M, Kaise S, Suzuki S, Kazuta Y, Sato Y, Miyata M, et al. Clinico-laboratory characteristics of patients with dermatomyositis accompanied by rapidly progressive interstitial lung disease. Clin Rheumatol. 1999;18:462-7.

3. Schnabel A, Reuter M, Biederer J, Richter C, Gross W. Interstitial lung disease in polymyositis and dermatomyositis: clinical course and response to treatment. Semin Arthritis Rheum. 2003;32:273-84.

4. Sato S, Hirakata M, Kuwana M, Suwa A, Inada S, Mimori T, et al. Autoantibodies to a 140-kd polypeptide, CADM-140, in Japanese patients with clinically amyopathic dermatomyositis. Arthritis Rheum. 2005;52:1571-6.

5. Ye S, Chen X, Lu X, Wu MF, Deng Y, Huang WQ, et al. Adult clinically amyopathic dermatomyositis with rapid progressive interstitial lung disease: a retrospective cohort study. Clin Rheumatol. 2007;26:1647-54.

6. Euwer RL, Sontheimer RD. Amyopathic dermatomyositis: a review. J Invest Dermatol. 1993;100:124S-7.

7. Kameda H, Nagasawa H, Ogawa H, Sekiguchi N, Takei H, Tokuhira M, et al. Combination therapy with corticosteroids, cyclosporin A, and intravenous pulse cyclophosphamide for acute/subacute interstitial pneumonia in patients with dermatomyositis. J Rheumatol. 2005;32:1719-26.

8. Saketkoo LA, Ascherman DP, Cottin V, Christopher-Stine L, Danoff SK, Oddis CV. Interstitial lung disease in idiopathic inflammatory myopathy. Curr Rheumatol Rev. 2010;6:108-19.

9. Connors GR, Christopher-Stine L, Oddis CV, Danoff SK. Interstitial lung disease associated with the idiopathic inflammatory myopathies: what progress has been made in the past 35 years? Chest. 2010;138:1464-74.

10. Labirua A, Lundberg IE. Interstitial lung disease and idiopathic inflammatory myopathies: progress and pitfalls. Curr Opin Rheumatol. 2010;22:633-8.

11. Nakashima R, Imura Y, Kobayashi S, Yukawa N, Yoshifuji H, Nojima T, et al. The RIG-H-like receptor IFIH1/MDA5 is a dermatomyositis-specific autoantigen identified by the anti-CADM-140 antibody. Rheumatology (Oxford). 2010;49:433-40. 
12. Gono T, Kawaguchi $Y$, Hara M, Masuda I, Katsumata $Y$, Shinozaki M, et al. Increased ferritin predicts development and severity of acute interstitial lung disease as a complication of dermatomyositis. Rheumatology (Oxford). 2010;49:1354-60.

13. Gono T, Kawaguchi $Y$, Satoh H, Kuwana M, Katsumata $Y$, Takagi K, et al. Clinical manifestation and prognostic factor in anti-melanoma differentiation-associated gene 5 antibody-associated interstitial lung disease as a complication of dermatomyositis. Rheumatology (Oxford). 2010;49:1713-9.

14. Gono T, Kawaguchi Y, Ozeki E, Ota Y, Satoh T, Kuwana M, et al. Serum ferritin correlates with activity of anti-MDA5 antibody-associated acute interstitial lung disease as a complication of dermatomyositis. Mod Rheumatol. 2011:21:223-7.

15. Bandoh S, Fujita J, Ohtsuki Y, Ueda Y, Hojo S, Tokuda M, et al. Sequential changes of KL-6 in sera of patients with interstitial pneumonia associated with polymyositis/dermatomyositis. Ann Rheum Dis. 2000;59:257-62.

16. Kubo M, Ihn H, Yamane K, Kikuchi K, Yazawa N, Soma Y, et al. Serum KL-6 in adult patients with polymyositis and dermatomyositis. Rheumatology (Oxford). 2000;39:632-6.

17. Satoh H, Kurishima K, Ishikawa H, Ohtsuka M. Increased levels of KL-6 and subsequent mortality in patients with interstitial lung disease. J Intern Med. 2006;260:429-34

18. Kumánovics G, Minier T, Radics J, Pálinkás L, Berki T, Czirják L. Comprehensive investigation of novel serum markers of pulmonary fibrosis associated with systemic sclerosis and dermato/polymyositis. Clin Exp Rheumatol. 2008;26:414-20.

19. Fathi M, Barbasso Helmers S, Lundberg IE. KL-6: a serological biomarker for interstitial lung disease in patients with polymyositis and dermatomyositis. J Intern Med. 2012;271:589-97.

20. He Z, Bateman A. Progranulin (granulin-epithelin precursor, PC-cell-derived growth factor, acrogranin) mediates tissue repair and tumorigenesis. J Mol Med. 2003;81:600-12.

21. Bateman A, Bennett HP. The granulin gene family: from cancer to dementia. Bioessays. 2009:31:1245-54

22. Daniel R, He Z, Carmichael KP, Halper J, Bateman A. Cellular localization of gene expression for progranulin. J Histochem Cytochem. 2000;48:999-1009.

23. He Z, Ong CH, Halper J, Bateman A. Progranulin is a mediator of the wound response. Nat Med. 2003:9:225-9.

24. Van Damme P, Van Hoecke A, Lambrechts D, Vanacker $P$, Bogaert E, van Swieten $J$, et al. Progranulin functions as a neurotrophic factor to regulate neurite outgrowth and enhance neuronal survival. J Cell Biol. 2008;181:37-41.

25. Yin F, Banerjee R, Thomas B, Zhou P, Qian L, Jia T, et al. Exaggerated inflammation, impaired host defense, and neuropathology in progranulindeficient mice. J Exp Med. 2010;207:117-28.

26. Kessenbrock K, Fröhlich L, Sixt M, Lämmermann T, Pfister H, Bateman A, et al. Proteinase 3 and neutrophil elastase enhance inflammation in mice by inactivating antiinflammatory progranulin. J Clin Invest. 2008;118:2438-47.

27. Park B, Buti L, Lee S, Matsuwaki T, Spooner E, Brinkmann MM, et al. Granulin is a soluble cofactor for Toll-like receptor 9 signaling. Immunity. 2011;34:505-13.

28. Tanaka A, Tsukamoto H, Mitoma H, Kiyohara C, Ueda N, Ayano M, et al. Serum progranulin levels are elevated in patients with systemic lupus erythematosus, reflecting disease activity. Arthritis Res Ther. 2012;14:R244.

29. Kim GT, Cho ML, Park YE, Yoo WH, Kim JH, Oh HJ, et al. Expression of TLR2, TLR4, and TLR9 in dermatomyositis and polymyositis. Clin Rheumatol. 2010;29:273-9.

30. Brunn A, Zornbach K, Hans VH, Haypt WF, Deckert M. Toll-like receptors promote inflammation in idiopathic inflammatory myopathies. J Neuropathol Exp Neurol. 2012;71:855-67.

31. Sun WC, Sun YC, Lin H, Yan B, Shi GX. Dysregulation of the type I interferon system in adult-onset clinically amyopathic dermatomyositis has a potential contribution to the development of interstitial lung disease. $\mathrm{Br} J$ Dermatol. 2012;167:1236-44.

32. Bilgic H, Ytterberg S, Amin S, McNallan KT, Wilson JC, Koeuth T, et al. Interleukin- 6 and type I interferon-regulated genes and chemokines mark disease activity in dermatomyositis. Arthritis Rheum. 2009;60:3436-46.33. Nara M, Komatsuda A, Omokawa A, Togashi M, Okuyama S, Sawada K, et al. Serum interleukin 6 levels as a useful prognostic predictor of clinically amyopathic dermatomyositis with rapidly progressive interstitial lung disease. Mod Rheumatol. 2014;24:633-6.
33. Haddad F, Zaldivar F, Cooper DM, Adams GR. IL-6-induced skeletal muscle atrophy. J Appl Physiol. 2005;98:911-7.

34. Okiyama N, Sugihara T, Iwakura Y, Yokozeki H, Miyasaka N, Kohsaka H. Therapeutic effects of interleukin- 6 blockade in a murine model of polymyositis that does not require interleukin-17A. Arthritis Rheum. 2009;60:2505-12.

35. Bohan A, Peter J. Polymyositis and dermatomyositis (first of two parts). N Engl J Med. 1975;292:344-7.

36. Sontheimer RD. Would a new name hasten the acceptance of amyopathic dermatomyositis (dermatomyositis siné myositis) as a distinctive subset within the idiopathic inflammatory dermatomyositis spectrum of clinical illness? J Am Acad Dermatol. 2002;46:626-36. A published erratum appears in J Am Acad Dermatol. 2002;46:699.

37. American Thoracic Society, European Respiratory Society. Idiopathic pulmonary fibrosis: diagnosis and treatment. International Consensus Statement. Am J Respir Crit Care Med. 2000;161:646-64.

38. Oryoji K, Himeji D, Nagafuji $K$, Horiuchi T, Tsukamoto $H$, Nondo $H$, et al. Successful treatment of rapidly progressive interstitial pneumonia with autologous peripheral blood stem cell transplantation in a patient with dermatomyositis. Clin Rheumatol. 2005;24:637-40.

39. Gono T, Miyake K, Kawaguchi Y, Kaneko H, Shinozaki M, Yamanaka H. Hyperferritinemia and macrophage activation in a patient with interstitial lung disease with clinically amyopathic DM. Rheumatology (Oxford). 2012;51:1336-8.

40. Kessenbrock K, Dau T, Jenne DE. Tailor-made inflammation: how neutrophil serine proteases modulate the inflammatory response. J Mol Med. 2011;89:23-8.

41. Tang W, Lu Y, Tian QY, Zhang Y, Guo FJ, Liu GY, et al. The growth factor progranulin binds to TNF receptors and is therapeutic against inflammatory arthritis in mice. Science. 2011;332:478-84.

42. Ihn H, Asano Y, Kubo M, Yamane K, Jinnin M, Yazawa N, et al. Clinical significance of serum surfactant protein D (SP-D) in patients with polymyositis/dermatomyositis: correlation with interstitial lung disease. Rheumatology (Oxford). 2002;41:1268-72.

43. Nakajima H, Harigai M, Hara M, Hakoda M, Tokuda H, Sakai F, et al. KL-6 as a novel serum marker for interstitial pneumonia associated with collagen disease. J Rheumatol. 2000;27:1164-70.

44. Matsubara T, Mita A, Minami K, Hosooka T, Kitazawa S, Takahashi K, et al. PGRN is a key adipokine mediating high fat diet-induced insulin resistance and obesity through IL-6 in adipose tissue. Cell Metab. 2012;15:38-50.

45. Jacob N, Stohl W. Cytokine disturbances in systemic lupus erythematosus. Arthritis Res Ther. 2011;13:228.

46. Papadimitraki ED, Choulaki C, Koutala E, Bertsias G, Tsatsanis C, Gergianaki I, et al. Expansion of Toll-like receptor 9-expressing B cells in active systemic lupus erythematosus: implications for the induction and maintenance of the autoimmune process. Arthritis Rheum. 2006;54:3601-11.

47. Jian J, Konopka J, Liu C. Insights into the role of progranulin in immunity, infection, and inflammation. J Leukoc Biol. 2013;93:199-208.

\section{Submit your next manuscript to BioMed Central and take full advantage of:}

- Convenient online submission

- Thorough peer review

- No space constraints or color figure charges

- Immediate publication on acceptance

- Inclusion in PubMed, CAS, Scopus and Google Scholar

- Research which is freely available for redistribution 